\title{
LA PREOCUPACIÓN POR LA NATURALEZA, ¿UN PROCESO NATURAL PARA LA POLÍTICA PÚBLICA? CÓMO PROMOVER QUE LOS TEMAS VINCULADOS CON EL DESARROLLO SOSTENIBLE SEAN CONSIDERADOS EN LAS AGENDAS AMBIENTALES DE LAS CIUDADES
}

María de los Ángeles Barrionuevo Mora*

\section{Resumen}

Ecuador es un país líder a nivel mundial por el reconocimiento de los derechos de la naturaleza y de la ciudad en su Constitución. Dado que Quito, su capital, se ha posicionado como uno de los pocos municipios que a nivel mundial han logrado acreditarse por cumplir compromisos internacionales en temas de cambio climático, se convierte en un caso relevante de estudio. A partir de la experiencia de este municipio se pretende identificar los principales factores que influyen para que un gobierno local asuma una agenda ambiental que procure la sostenibilidad. Aunque los contextos nacional e internacional intervienen para posicionar estos temas, factores como el liderazgo, la influencia de redes internacionales, el nivel de especialización del aparato burocrático y la normatividad local han permitido promover los temas vinculados con el desarrollo sostenible a nivel local

* Doctorado en Políticas Públicas, Facultad Latinoamericana de Ciencias Sociales (flacso), Quito Ecuador. Docente, Facultad de Economía, Pontificia Universidad Católica del Ecuador (PUCE). Quito (Ecuador). [mabarrionuevom@puce.edu.ec]

Recibido: 1 de julio de 2016 / Modificado: 31 de agosto de 2016 / Aceptado: 1 de septiembre de 2016

Para citar este artículo

Barrionuevo Mora, M. A. (2016). La preocupación por la naturaleza, ¿un proceso natural para la política pública? Cómo promover que los temas vinculados con el desarrollo sostenible sean considerados en las agendas ambientales de las ciudades. OPERA, 19, pp. 35-53.

DOI: http://dx.doi.org/10.18601/16578651.n19.04 
y así visibilizar a las ciudades como actores relevantes para la procura de un modelo de sostenibilidad.

Palabras clave: Ecuador, Distrito Metropolitano de Quito, agenda de sostenibilidad, desarrollo sostenible, políticas públicas, adopción de agenda.

\section{THE CONCERN FOR NATURE, A NATURAL PROCESS FOR PUBLIC POLICY? HOW TO PROMOTE SUSTAINABLE DEVELOPMENT IN URBAN ENVIRONMENTAL AGENDAS}

\section{Abstract}

Ecuador is a leader worldwide for its recognition of the Rights of Nature and the Rights of the City in its Constitution. In so far as Quito, its capital, has positioned itself among the first municipalities that have demonstrated their compliance with the international commitments on climate change, it is a relevant case study. From the experience of this city, we aim to identify the main factors that contribute to local government taking on a sustainability agenda. Although the national and international context influence on the positioning of such topics, such factors as leadership, the influence of international networks, the level of specialization of the bureaucratic apparatus and local regulations have permitted to include issues related to sustainable development in the public policy agenda and thus have given visibility to cities as relevant actors in the pursuit of sustainable development.
Key words: Ecuador, Quito Metropolitan District, sustainable agenda, sustainable development, public policies, agenda setting.

\section{INTRODUCCIÓN}

El enfoque secuencial de análisis de las políticas públicas ubica a la definición y adopción de la agenda como la primera etapa del ciclo y es en esta en la que se determinan los problemas que serán considerados por los hacedores de política pública (Green-Pedersen y Mortensen, 2013, p. 167). La agenda ha sido conceptualizada como "una colección de problemas, comprensión de las causas, símbolos, soluciones y otros elementos de un problema público que capturan la atención de los miembros del sector público y de sus funcionarios" (Fischer y Miller, 2007, p. 63), así como de aquellos que se encuentran fuera del Gobierno pero que son cercanos a este y pueden influir en el proceso de toma de decisiones (Kingdon, 2003, p. 3). El análisis de la agenda pública permite comprender cómo una situación se convierte en una condición que debe ser atendida desde lo público y cómo, a partir de ello, escala para convertirse en un problema público. Esto permite explicar por qué ciertos temas son priorizados sobre otros y logran posicionarse en la agenda de política pública.

Fischer y Gottweis (2012, pp. 43-44) establecen que, cuando la sociedad se interesa por un tema y comienza a demandar su resolución, los problemas públicos son enunciados y se empieza a definir la agenda de política pública. A nivel ambiental, al final de la década 
de los setenta Cockburn y Ridgeway (1979) vincularon el concepto de la degradación ambiental con aspectos políticos, y ello dio paso a una reflexión sobre la vinculación de los procesos económicos y políticos con el uso de los recursos naturales. Esto permitió establecer que las relaciones sociedad-ambiente no son apolíticas, y reconocer que "lo político es inevitablemente ecológico y que lo ecológico es inherentemente político" (Robbins, 2012, p. 3).

A nivel ambiental, el abordaje urbano es de gran relevancia. Según proyecciones estadísticas del Fondo de Población de las Naciones Unidas (UNFPA), la región de América Latina y el Caribe tendrá una población urbana cercana al $90 \%$ en el año 2050 (UNFPA, 2007). Sin embargo, además del crecimiento poblacional, la urbanización puede generar otros problemas ambientales tales como los procesos migratorios; las modalidades de planificación; la institucionalidad y gobernabilidad que se ejercen en las ciudades; así como las políticas y los programas que se definen para enfrentar la problemática de lo urbano (Heynen, Kaika y Swyngedouw, 2006, p. 6). Así, la problemática ambiental incluye desde los temas de saneamiento hasta los que procuran un modelo de sostenibilidad.
Algunas condiciones urbanas pueden producirse y reproducirse en luchas generadas por enfrentamientos socioecológicos que se originan en las relaciones sociales y materiales y en su vinculación con la naturaleza (Jahn, 1991, en Heynen, Kaika y Swyngedouw, 2006, p. 1). Por tanto, lo que debemos preguntarnos es quién produce qué tipos de configuraciones ecológicas y para quién lo hace. La respuesta a este cuestionamiento está directamente vinculada con la forma en la cual se configura una agenda ambiental urbana.

El Ecuador ha sido reconocido como un país megadiverso por su riqueza biológica; no obstante, tener una economía altamente dependiente de sus recursos naturales puede comprometer su existencia. Por la importancia de estos recursos, la política pública ambiental debería haber sido priorizada a nivel nacional y local pero el tema no ha logrado aún posicionarse. A fin de comprender cuáles son los elementos que han influido para que las ciudades decidan priorizar ciertos temas sobre otros se ha tomado como caso de estudio la agenda ambiental urbana del Distrito Metropolitano de Quito (DMQ) ${ }^{1}$. Esta ciudad resalta a nivel nacional e internacional por el nivel de compromiso asumido en acuerdos y convenios vinculados con temas de sosteni-

1 Quito es la capital del Ecuador. "El Distrito Metropolitano de Quito está localizado en la provincia de Pichincha y su mayor parte se ubica en la Cordillera Occidental de los Andes, en las cuencas del río Guayllabamba y alta del río Esmeraldas; ocupa una superficie aproximada de 423.000 ha, dentro de un rango altitudinal que va desde los 500 a los $4790 \mathrm{msnm}$. [...] El amplio rango altitudinal y las condiciones orográficas han permitido que en el DMQ se desarrollen 6 clases de formaciones vegetales divididas en 17 subclases que van desde los bosques húmedos a bosques secos, los arbustos y herbazales húmedos y secos. De igual forma se pueden distinguir 15 tipos de clima, desde el tropical lluvioso en zonas bajas hasta el páramo y nivel en las zonas más altas" (PNUMA, FAMDMQ, FLACso, 2011, p. 21). 
bilidad y cambio climático. Sobre la base de información bibliográfica recabada se realizará un recorrido histórico para identificar la manera en la cual los temas relacionados con un modelo que procura la sostenibilidad se han ido incorporando en el quehacer de su política pública.

En este documento se propone contribuir a la reflexión acerca de los factores que influyen para que los temas vinculados con el desarrollo sostenible se impongan en la agenda de política pública a nivel local.

\section{EL SURGIMIENTO DE LA PREOCUPACIÓN POR LA PROBLEMÁTICA AMBIENTAL A NIVEL LOCAL EN ECUADOR}

El interés por el tema ambiental a nivel local surge por la influencia de la política ambiental internacional producto de la creciente preocupación por la relación del ser humano con el ambiente y de los impactos que estos generan en la naturaleza. En los años setenta emerge una manifestación de descontento social en la que a partir de avances científicos y de la movilización simbólica se intentaba probar que el mundo y la vida estaban en peligro de extinción (Lezama, 2008, p. 14).

En 1972 se producen dos eventos que vendrían a constituir el punto de partida de la política ambiental internacional y que incidirían en el abordaje de la temática ambiental en el Ecuador y a nivel local: el Informe del Club de Roma -conocido también como el Informe Meadows- y la Conferencia de Estocolmo. El informe hacía énfasis en que el planeta estaba llegando a los límites de creci- miento y que era necesario que los países aunaran esfuerzos para enfrentar esta situación. Ecuador, país firmante de la Declaración de Estocolmo, comprometió sus esfuerzos a la institucionalización ambiental, la definición de políticas públicas, la consolidación de los sistemas jurídicos y la estructuración del aparataje público en el Ecuador (Fontaine, Narváez y Cisneros, 2008).

En 1974 se realizó la Conferencia de Cocoyoc, en México, en la que se firmó la Carta de Deberes y Derechos de los Estados y se planteó que los "Estados deben tratar de establecer sus propias políticas ambientales y de desarrollo de conformidad con esa responsabilidad. Las políticas ambientales de todos los Estados deben promover y no afectar adversamente el actual y futuro potencial de desarrollo de los países en vías de desarrollo" (Declaración de Cocoyoc, 1974, art. 30, en CEPAL, 2016).

En el año 1987 se presentó el informe Brundtland -conocido también como Nuestro Futuro Común-y con ello se motivó el debate sobre la necesidad de procurar un modelo de desarrollo sostenible que permita satisfacer las necesidades del presente sin comprometer las de futuras generaciones. Como respuesta a ello, en el país también se empezó a reflexionar sobre la necesidad de un nuevo modelo que procure el bienestar de las actuales y de las futuras generaciones, y en el cual se priorice lo que el ser humano puede "'hacer y ser' más que en lo que puede tener" (Larrea, 2012, p. 27). El modelo se trasladó como un tema de preocupación local y en 1989 se ejecutó en Quito el primer proyecto de evaluación ambiental de contaminantes atmosféricos, 
hídricos e industriales, y en 1990 se creó el Departamento de Control de Calidad Ambiental en la Dirección de Higiene.

A partir de la Conferencia de Río en 1992, con la firma de la Declaración de Río sobre el Medio Ambiente y el Desarrollo conocida como la Agenda 21, el concepto de desarrollo sostenible empezó a trascender. El capítulo $28^{2}$ de dicha Agenda reconoce la necesidad de colaborar y ser responsables, a todos los niveles, para alcanzar el desarrollo sostenible reconociendo así la importancia del involucramiento de los gobiernos locales. Como respuesta a ello se plantea la Agenda Local 21 en la cual se hace énfasis en el rol que las autoridades locales tienen en su promoción. Como respuesta a ello se forma el Local Governments for Sustainability (ICLEI), una asociación internacional de gobiernos locales que decidieron involucrase en el desarrollo sostenible (ICLEI, 2015).

En el año 1993, los países suscribieron el Convenio de Diversidad Biológica (CDB) comprometiéndose a garantizar el derecho de las personas a vivir en un ambiente libre de contaminación y poner de manifiesto el deber que el Estado debía asumir para velar por su cumplimiento y procurar la preservación de la naturaleza (Albán, Barragán, Bedón y Muñoz, 2011). Los compromisos adquiridos en el CDB se trasladaron a todo nivel territorial aunque su aplicación estaba sujeta a las atribuciones conferidas a cada nivel de gobierno.

Como parte del proceso de posicionamiento de los temas ambientales a nivel local, en 1994 se creó la Dirección Metropolitana de Medio Ambiente, actualmente conocida como Secretaría de Ambiente, y el municipio de Quito solicitó su membresía al ICLEI. En 1998 se crearon "dos entidades clave para el desarrollo de la gestión ambiental: la Comisión de Medio Ambiente y Riesgos Naturales del Concejo Metropolitano, y la Comisaría Ambiental" (PNUMA, FAMDMQ, FLACSO, 2011, p. 51).

A nivel de país se impulsó el tratamiento de la temática ambiental a través del estable-

\footnotetext{
2 "El capítulo 28 propone una serie de actuaciones programadas en el ámbito local para que el desarrollo sea globalmente sostenible. Insta a los poderes locales a iniciar un proceso de diálogo con sus ciudadanos, organizaciones y entidades para adoptar un plan de desarrollo centrado en las oportunidades y valores locales. La clave radica en el hecho de considerar de una manera integrada el desarrollo social, el económico y el medio ambiente, y por tanto aborda temas que tradicionalmente se han tratado de una manera estanca.

Las medidas que se proponen intentan detener la destrucción del medio ambiente y eliminar las desigualdades entre los países.

Los objetivos se concretan en la lucha contra la pobreza, la protección y el fomento de la salud, la protección de la atmósfera, la conservación y el uso racional de los recursos forestales, la lucha contra la desertización, la protección de los ecosistemas de montaña, el desarrollo de la agricultura sin agredir al suelo, la conservación de la biodiversidad, la gestión racional y ecológica de la biotecnología, la protección de los recursos oceánicos y de agua dulce, la seguridad en el uso de los productos tóxicos y la gestión de los desechos sólidos, peligrosos y radiactivos [...].

La Cumbre de la Tierra marca un hito histórico: la instauración de un nuevo sistema de entendimiento mundial para el desarrollo sostenible, basado en la indivisibilidad de la protección ambiental y el proceso de desarrollo" (Agenda 21 local, s. f., p. 30).
} 
cimiento de las políticas ambientales básicas generales en el año 1994, así como la creación del Ministerio del Ambiente (MAE) y la elaboración del Plan Ambiental Ecuatoriano en el año 1996 (Fontaine, Narváez y Cisneros, 2008). A raíz de la firma del CDB surgen los Fondos Ambientales, mecanismos financieros que actúan para facilitar la implementación de las políticas y acciones de conservación y uso sostenible de la biodiversidad. Estos fondos promueven la consecución de las 20 Metas Aichi para la Biodiversidad adoptadas en el marco del CDB en el año 2010 (REDLAC, 2016). El municipio de Quito ha sido una de las ciudades líderes en este proceso, pues en el año 1997 se creó el Fondo Ambiental para la Protección de las Cuencas y Agua (FONAG). El éxito del modelo permitió expandir la idea a otras ciudades latinoamericanas demostrando así el liderazgo de esta municipalidad en la temática (FONAG, 2016).

\section{¿EL BUEN VIVIR, UNA PROPUESTA PARA OPERATIVIZAR EL DESARROLLO SOSTENIBLE?}

Los cambios y las nuevas propuestas que se estaban gestando abrieron paso a un conjunto normativo ambiental que fue plasmado en la Constitución de 1998, en la cual se evidenciaba un punto de inflexión a partir del cual se va gestando la nueva política ambiental y que permitió avanzar en los derechos humanos de tercera y cuarta generación. Principalmente, se logró el reconocimiento de los derechos colectivos de los pueblos indígenas y afroecuatorianos, así como los derechos colectivos de los ciudadanos a la protección del medio ambiente.

Lo que es importante recalcar es que el impulso al tratamiento ambiental no se dio por la existencia de un movimiento social organizado en torno a la temática ambiental, sino que fue producto de las demandas formuladas por los movimientos sociales indígenas. Aunque la Constitución del año 1998 es un hito histórico desde el punto de vista ambiental, no podemos establecer que sea el punto de partida de la política ambiental pues esta ya existía, aunque se encontraba desarticulada del pensamiento constitucionalista. Con la entrada en vigencia de la Constitución del 98 , se constitucionaliza la materia ambiental, se viabiliza la tipificación de los delitos ambientales y la legislación ambiental se convierte en uno de los pilares de la política ambiental. La Constitución fortalece el rol del Estado en la procura del desarrollo sostenible, pero no se hacía mención a la manera de proceder en temas de planificación ambiental.

En términos ambientales podemos establecer que la Constitución de 1998 es una transición hacia los derechos que se consolidarían diez años después y que se profundizan en la Constitución del 2008, la cual es reconocida como una de las más progresistas a nivel mundial al incluir los Derechos de la Naturaleza ${ }^{3}$,

3 Los derechos de la naturaleza sientan un precedente internacional pues dejan de ver a la naturaleza como un recurso para concebirla como el espacio donde se reproduce y realiza la vida. Desde esta concepción, la naturaleza 
los Derechos Comunitarios ${ }^{4}$ y el Derecho a la Ciudad 5 , y tener una legislación secundaria que fortalece los principios constitucionales.

La Constitución del 2008 pone de manifiesto la necesidad de cambiar la relación sociedad-ambiente-Estado y recuperar "una visión de desarrollo que privilegia la consecución del buen vivir, que presupone la ampliación de las libertades, oportunidades y potencialidades de los seres humanos, y el reconocimiento de unos a otros para alcanzar un porvenir compartido" (senplades, 2015). Aunque se han generado varios debates con respecto a la propuesta del Buen Vivir ${ }^{6}$, el alcance que tiene para la definición de la política pública a nivel nacional y local no es cuestionable pues reveló la necesidad de crear una nueva organización política-administrativa del Estado ecuatoriano en el territorio a partir del cual se propuso un nuevo modelo de descentralización ${ }^{7}$.

Se establece que el ejercicio de la tutela sobre el ambiente se realizaría a través de una gestión concurrente y subsidiaria de las competencias (art. 136 del COOTAD) y que

3 Los derechos de la naturaleza sientan un precedente internacional pues dejan de ver a la naturaleza como un recurso para concebirla como el espacio donde se reproduce y realiza la vida. Desde esta concepción, la naturaleza tiene "derecho a que se respete integralmente su existencia y el mantenimiento y regeneración de sus ciclos vitales, estructura, funciones y procesos evolutivos", así como el derecho a la restauración (arts. 71 y 72); el principio de precaución (arts. 73 y 396); la no apropiación de los servicios ambientales (art. 74); la prohibición de realización de actividades extractivistas en áreas protegidas (art. 407); se reconoce el derecho de las comunidades a ser consultadas antes de la aprobación de cualquier decisión que pudiera afectar al medio ambiente y se reconoce el derecho de los pueblos ancestrales de mantener, proteger y desarrollar sus conocimientos colectivos, ciencias, tecnologías y saberes, y se prohíbe toda forma de apropiación de los mismos (art. 57).

4 Garantizan la consulta comunitaria para la explotación de recursos no renovables.

5 "Las personas tienen derecho al disfrute pleno de la ciudad y de sus espacios públicos, bajo los principios de sustentabilidad, justicia social, respeto a las diferentes culturas urbanas y equilibrio entre lo urbano y lo rural. El ejercicio del derecho a la ciudad se basa en la gestión democrática de esta, en la función social y ambiental de la propiedad y de la ciudad, y en el ejercicio pleno de la ciudadanía" (art. 31, Constitución de 2008).

6 El término empezó a posicionarse a partir del año 2000, cuando en Bolivia la Agencia de Cooperación Alemana (GTZ) realizó un programa especial llamado "Suma Qamaña", cuyos principios de vida austera en armonía y equilibrio a nivel personal, comunitario y con el cosmos comenzaron a ser difundidos a través de publicaciones y seminarios que permearon en países como el Ecuador en el cual se lo asimiló bajo el concepto indígena del "Buen Vivir" (alli kawsay) (Altmann, 2013, p. 291-292).

7 A partir de la Constitución del 2008 se conformó el Consejo Nacional de Competencias y se estableció el Código Orgánico de organización Territorial, Autonomía y Descentralización (COOTAD) publicado en el Suplemento 303 del Registro Oficial del 19 de octubre del 2010, a través del cual se procura mejorar el ejercicio de los derechos ciudadanos establecidos en la Constitución. La competencia de gestión ambiental se enmarca, entre otros, en el artículo 399 de la Constitución en el que se establece que "el ejercicio integral de la tutela estatal sobre el ambiente y la corresponsabilidad de la ciudadanía en su preservación, se articulará a través de un sistema nacional descentralizado de gestión ambiental, que tendrá a su cargo la defensoría del ambiente y la naturaleza”, y del artículo 125 del COOTAD que plantea que "los gobiernos autónomos descentralizados son titulares de nuevas competencias exclusivas constitucionales las cuales se asumirán e implementarán de manera progresiva conforme lo determine el Consejo Nacional de Competencias". 
la descentralización ambiental deja de ser voluntaria y pasa a ser obligatoria. A fin de implementar los principios constitucionales, la Secretaría Nacional de Planificación y Desarrollo (SENPLADES) propuso un documento de planificación denominado Plan Nacional de Desarrollo o Plan Nacional del Buen Vivir (PNBV) en el cual se plasmaron cambios en la conceptualización de la problemática ambiental desde la jurisdicción territorial y administrativa y que sería posteriormente asumida en todo proceso de planificación.

En la regulación de la competencia de gestión ambiental se definieron las competencias de los gobiernos autónomos descentralizados provinciales, metropolitanos, municipales y parroquiales rurales reconociendo sus facultades con respecto a la rectoría, planificación, regulación, control y gestión en su territorio. Las atribuciones y competencias son plasmadas en el Código Orgánico de Organización Territorial, Autonomía y Descentralización $(\text { COOTAD })^{8}$, documento en el cual se definieron las competencias temáticas a nivel territorial. La nueva propuesta superó a la Ley de Descentralización anterior, ya que en esta "no se diferenciaban las atribuciones por nivel de gobierno (nacional, provincial y municipal), lo que derivó, [...] en ambigüedades y superposiciones" (Krainer y Mora, 2013, p. 40). En el nuevo modelo, la transferencia de competencias es diferenciada en cada nivel de Gobierno para optimizar la administración (art. 2, СOOTAD). El documento permite visibilizar que los temas ambientales son multidimensionales y que en muchas ocasiones la resolución de un problema demanda el involucramiento de diferentes niveles de gobierno, por lo que se requiere una adecuada planificación y gestión.

El artículo 55 del COOTAD determina que entre las competencias exclusivas del gobierno autónomo descentralizado municipal, objeto de este análisis, se encuentran el

...prestar los servicios públicos de agua potable, alcantarillado, depuración de aguas residuales, manejo de desechos sólidos, actividades de saneamiento ambiental y aquellos que establezca la ley; [...] delimitar, regular, autorizar y controlar el uso de las playas de mar, riberas y lechos de ríos, lagos y lagunas, sin perjuicio de las limitaciones que establezca la ley; [...] preservar y garantizar el acceso efectivo de las personas al uso de las playas de mar, riberas de ríos, lagos y lagunas; [...] regular, autorizar y controlar la explotación de materiales áridos y pétreos, que se encuentren en los lechos de los ríos, lagos, playas de mar y canteras.

Así se puede apreciar que el concepto del Buen Vivir fue incorporándose al modelo de descentralización a fin de que cada nivel territorial aporte en el proceso de su consecución. En el caso del municipio de Quito se busca impulsar colectivamente el nuevo régimen de desarrollo, actuando de manera creativa, con sentido de construcción ciudadana e institucional (PNUMA, FAMDMQ, FLACSO, 2011, p. 30). En este escenario se promueve

$8 \quad$ Establecida en el Acuerdo 0005-CNC-201 y publicada en el Registro Oficial 415 el 13 de enero del 2015. 
un nuevo modelo de desarrollo que parte de una "racionalidad ambiental que incluye lo empírico, pasa por lo simbólico y apela hasta lo impensado, en función de prever y adaptarse a nuevas realidades" (p. 29).

El nuevo modelo se refuerza también a través de los derechos a la ciudad propuestos en la Constitución del 2008, a través de los cuales se establece la necesidad de promover una ciudad verde que sea limpia y amigable con el ambiente, que cuente con una movilidad inteligente en la cual se priorice a las personas y no a los automóviles, una ciudad segura y solidaria, educadora saludable e incluyente, que promueva equidad territorial, y una ciudad que gestione el espacio público a la vez que promueva la cultura (PNUMA, FAMDMQ, FLACSO, 2011, p. 19).

Entre las prioridades que se plantean en el marco del nuevo modelo está la adecuada gestión de agua, aire, suelo, biodiversidad y energía, mejorar la gestión de residuos sólidos y enfrentar el cambio climático. Esto invita a que se actúe teniendo como objetivo central el cambio de los patrones de producción y consumo, así como de las maneras en las cuales se debe realizar la apropiación del territorio $\mathrm{y}$ de los recursos naturales (PNUMA, FAMDMQ, FLACSO, 2011, p. 29). Así, se esperaría que la gente, en libertad y racionalidad, elija producir $\mathrm{y}$ consumir bienes que sean ambientalmente amigables, aunque para ello se debe construir la capacidad y lograr que los agentes interioricen estos esquemas de comportamiento (De Munck, 2014, pp. 31-32).

\section{LA INCLUSIÓN DE UN ENFOQUE DE SOSTENIBILIDAD EN LA AGENDA DE POLÍTICA PÚBLICA DEL DISTRITO METROPOLITANO DE QUITO}

Larrea (2012, p. 13) menciona que "cada periodo histórico se configura por una serie de relaciones sociales que reflejan unos imaginarios, unos modos de vida, unos valores y unas formas de organización de la vida en sociedad”. Así, para entender cuál ha sido la relación entre la política pública local y el modelo de sostenibilidad es importante comprender el "contexto histórico, social y ambiental que se forma continuamente por las luchas políticas, los cambios en el medio ambiente y los valores de la naturaleza" (Miller, 2007, en De Castro, Hogenboom y Baud, 2015 , p. 24), así como la manera en la cual se establecen las modalidades de coordinación.

La agenda de política pública del municipio del Distrito Metropolitano de Quito es un claro ejemplo de su compromiso con el modelo de desarrollo sostenible. Quito es una de las cuatro ciudades en el Ecuador que tienen información documentada sobre la calidad del aire, pero de estas solo Quito posee una red automatizadas de monitoreo atmosférico y cuenta con una Corporación para el Mejoramiento del Aire (CORPAire), la cual reporta la calidad del aire de la ciudad permanentemente (FLACSO, MAE, PNUMA, 2008), esto ha permitido determinar que el $80 \%$ de la contaminación del aire sea atribuida al parque automotor.

La contaminación sonora es otro tema al que Quito presta atención. La ciudad cuenta con 65 puntos de monitoreo en los cuales se 
reporta que el $25 \%$ de las administraciones zonales superan los $70 \mathrm{db}(\mathrm{A})$ y $80 \mathrm{~dB}(\mathrm{~A})$, que es el valor referencial límite para el ruido de las ciudades; la contaminación se debe principalmente al incremento del parque automotor (PNUD-CISMIL-MDMQ, 2008, p. 205).

Con respecto al recurso hídrico, Quito cuenta con información sobre contaminación de los principales ríos (Machángara en el que se descargan el $75 \%$ de las aguas residuales y Monjas que recibe el $20 \%$ de las aguas residuales de la ciudad) y realiza el manejo de cuencas hidrográficas municipales. Adicionalmente, cuenta con una Empresa Pública Metropolitana de Agua Potable y Saneamiento (EPMAPS) a través de la cual se ha logrado dar cobertura de agua potable al 99,63\% de la población y el $96,41 \%$ cuenta con servicio de alcantarillado.

Con respecto a la gestión de desechos la ciudad también cuenta con la Empresa Pública Metropolitana de Aseo (emaseo), la cual se ocupa de prestar el servicio de aseo y recolección de residuos sólidos ordinarios y peligrosos, y mejorar las prácticas ambienta- les mediante el proceso 4R (rechaza, reduce, reúsa y recicla). Los informes manifiestan que el nivel de cobertura efectiva de recolección estimado por EMASEO es del $96 \%$ en la ciudad (Empresa Pública Metropolitana de Aseo EMASEO, 2010).

Estos indicadores revelan que los temas marrones y grises en la ciudad de Quito están prácticamente, cubiertos por lo que esto llevaría a pensar que cuenta con las condiciones idóneas para preocuparse por incluir en sus políticas públicas los temas relacionados con un modelo de desarrollo sostenible. Así, es necesario reflexionar desde cuándo empieza la preocupación del municipio por estos temas.

La Dirección Metropolitana de Medio Ambiente (DMMA) del municipio de Quito fue creada en 1994, pero es en la administración de Paco Moncayo (2000-2009) ${ }^{9}$ en la que se consolida la gestión ambiental del DMQ y se formula el Plan Maestro de Gestión Ambiental (PMGA) ${ }^{10}$ en el año 2000 (Vallejo, 2009, pp. 97-99). Entre los objetivos que contiene el plan se pueden mencionar el mejorar la calidad del aire, agua, suelo, protección de los recur-

\footnotetext{
9 Paco Moncayo fue presidente del UCGL y durante su administración (2000-2004) se elaboraron importantes documentos para la planificación de la ciudad, entre los que resalta el Plan Quito siglo xxi. En el siguiente periodo de Gobierno (2004-2009) se presentó el Plan Estratégico Equinoccio 21 (Quito hacia el 2025) y el plan de Gobierno Quito hacia el Bicentenario 2005-2009, en el que se resaltó como uno de los ejes estratégicos el territorial, que procuraba entre otros temas la sostenibilidad ambiental así como el eje de gobernabilidad e institucionalidad (PNUMA, FAMDMQ, FLACSO, 2011, p. 223). Adicionalmente, se aprobaron las ordenanzas 094 (septiembre de 2003), 12 (diciembre de 2004), 146 (agosto de 2005) y 213 (septiembre de 2007).

10 Promovido por el alcalde Roque Sevilla y el director de Medio Ambiente del municipio Jorge Jurado. Roque Sevilla fue uno de los fundadores de Fundación Natura, organización no gubernamental creada en 1978 y que fue la primera institución ambientalista activa que trató de impulsar propuestas legales (Albán, Barragán, Bedón y Muñoz, 2011, p. 26). Sevilla ejerció la presidencia de Fundación Natura por diez años, fue miembro de la Asamblea General de la Fundación Charles Darwin, ejerció como director de la Fundación Esquel y fue miembro del Fondo Mundial para la Naturaleza (wwF).
} 
sos naturales y la biodiversidad y propender hacia una ciudad verde. A finales de 2004 se realizó el traslado de competencias desde el MAE al municipio que permitieron fortalecer las acciones que venía realizando.

Posteriormente, se generó una nueva versión revisada y actualizada del Plan para el periodo 2004-2010, donde se incluyeron las estrategias, los objetivos y las metas de mejoramiento de la calidad ambiental y se aclararon las directrices para operativizarlas.

En la administración de Augusto Barrera (2009-2013) se elaboró la Agenda ambiental 2011-2016 ${ }^{11}$, en la que se plantea, entre otros temas, la necesidad de manejar sustentablemente la riqueza natural, prepararse y responder oportunamente al cambio climático y construir un Quito verde (Alcaldía del Distrito Metropolitano de Quito, 2011). Adicionalmente, se publicó la Estrategia Ambiental 2010-2015, en la cual se establecieron como prioridades:

...fortalecer la autoridad ambiental bajo los principios de gobernabilidad, sostenibilidad y eficiencia; convertir al Distrito Metropolitano de Quito en un modelo de gestión sustentable y eficiente de la gestión ambiental; promover el cambio de matriz energética; convertir al Distrito Metropolitano de Quito en una ciudad pionera en respuestas al cambio climático; promover la conservación de la biodiversidad y el manejo integrado de ecosistemas; reducir la contaminación atmosférica (PNUMA, FAMDMQ, FLACSO, 2011, p. 50).

Actualmente, el municipio cuenta con una Secretaría de Ambiente, la cual cuenta con tres direcciones: Dirección Metropolitana de Políticas y Planeamiento Ambiental; Dirección Metropolitana de Gestión del Patrimonio Nacional; y, Dirección Metropolitana de la Calidad Ambiental. Las dos primeras direcciones tienen una línea de trabajo vinculada con los temas de la agenda de sostenibilidad pues ampara a la Unidad de cambio climático, Unidad de biodiversidad y ecosistemas, Unidad de gestión de áreas protegidas y la Unidad de recuperación y monitoreo de la cobertura vegetal, en tanto que la tercera se ocupa más de los temas grises y marrones. Adicionalmente, el municipio cuenta con el Subsistema Metropolitano de Áreas Naturales Protegidas del Distrito Metropolitano de Quito que procura la conservación de los ecosistemas más frágiles y amenazados.

De forma paralela surgieron espacios de colaboración internacional para apoyar el cumplimiento de los acuerdos de Río 92, a los que el municipio de Quito se fue vinculando directa o indirectamente. En el año 1994 se llevó a cabo en Dinamarca la Conferencia Europea de Ciudades y Pueblos Sostenibles, en la que las ciudades europeas manifestaron su compromiso con la Agenda Local 21 y firmaron la Carta de Aadolborg. A partir de ello se reunieron en 1996, en Lisboa (Segunda Conferencia Europea de Ciudades y Pueblos Sostenibles), cerca de 1000 autoridades municipales y regionales de 35 países europeos a fin de acordar la necesidad de implementar la carta de Aadolborg. En el mismo año, Naciones Unidas organizó en Estambul la Conferencia

11 Promovida por el alcalde Augusto Barrera y el secretario de Ambiente del Municipio Ramiro Morejón Neira. 
sobre Asentamientos Humanos (Habitat II). En el año 1999, en España, se llevó a cabo la Conferencia Euro-Mediterránea de Ciudades Sostenibles. En el año 2000 se realizó en Alemania la Tercera Conferencia Europea de Ciudades y Pueblos Sostenibles (Agenda 21 Local, 1992, pp. 28-29). Estos espacios permitieron fortalecer la capacidad institucional de los gobiernos locales y sentaron la base de las alianzas a las que posteriormente Quito se vincularía.

A partir del año 2000 surgió una segunda ola de acción municipal en temas ambientales, especialmente vinculados con la temática de cambio climático y la gobernanza climática urbana. En el año 2004 se creó la Red Mundial de Ciudades y Gobiernos Locales y Regionales (UCLG) a fin de influenciar en la agenda global de desarrollo y "construir un mundo pacífico basado en el desarrollo sostenible" (UCLG, 2016). Un elemento importante por considerar es que la representación para América Latina y El Caribe de esta organización es la Federación Latinoamericana de Ciudades, Municipios y Asociaciones de Gobiernos Locales (FLACMA), la cual "tiene sus raíces en lo que fue el Capítulo Latinoamericano de la Unión Internacional de Administradores Locales, IULA [...] [y] se fundó el 17 de noviembre de 1981 con sede en la ciudad de Quito, República del Ecuador" (FLACMA, 2015).

Un año después se creó el C40, en el marco del cual se establece el Consejo Mundial de Alcaldes sobre Cambio Climático que "fue fundado en diciembre de 2005 por Yorikane Masumoto, alcalde de la ciudad de Kyoto, poco después de que el Protocolo de Kyoto entró en vigor" (World Mayors
Council on Climate Change, 2015). El municipio de Quito se afilió a esta red en el año 2015 y es el único municipio del Ecuador adscrito a la coalición conformada por 83 ciudades a nivel mundial.

En la cop 13 (2007) se firmó la hoja de ruta de Bali y los representantes de los municipios firmaron el Acuerdo Mundial de los Alcaldes y Gobiernos Locales sobre la Protección del Clima (C40 de Bali). En este evento se lanzó la Hoja de Ruta Climática de los Gobiernos Locales durante las sesiones de los Gobiernos Locales sobre el Clima celebradas paralelamente a la cop13 (Barcelona Climate Change Talks, 2009). Aunque Ecuador es signatario y participa en las reuniones de la COP, la participación en este evento fue con una delegación nacional y no local.

En el año 2010 se firmó el Pacto de la Ciudad de México como una muestra por parte de las ciudades de su compromiso de enfrentar y trabajar para mitigar el cambio climático, el municipio de Quito se hizo signatario al Pacto el 21 de noviembre de 2010. En el mismo año, los gobiernos locales lanzaron el Registro Climático de Ciudades carbonn (cCCR por sus siglas en inglés), que es una base de datos mundial que permite obtener información medible para ser empleada en la planeación de los gobiernos locales y Quito se adscribió a este compromiso. El municipio de Quito, ratificando su acuerdo de liderar la lucha frente al cambio climático en Ecuador y América Latina, asumido en México, realizó en junio del 2011 la Primera Cumbre Nacional de Autoridades Locales, cuyo objetivo fue lograr compromisos desde lo local a fin de desarrollar políticas públicas y normativas para 
la gestión del cambio climático. Así, a partir de la incidencia de este municipio se dio paso a la firma del "Pacto climático de Quito" por parte de los municipios y gobiernos provinciales ecuatorianos que asistieron al evento.

En el año 2013 se llevó a cabo la Cumbre Mundial de Alcaldes sobre el Cambio Climático, en la cual se adoptó la Declaración de Nantes de Alcaldes y Líderes Subnacionales en Cambio Climático. En el año 2014 se realizó la Cumbre del Clima, en la que las redes globales C40, ICLEI, UCLG y otros aliados crearon la mayor coalición de gobiernos locales para enfrentar el cambio climático denominada Compact of Mayors (UCLG, 2015). Esta coalición surgió como preparación para la COP 21, realizada en París en el 2015, con el objetivo de promover la reducción de gases de efecto invernadero (GEI), mejorar la resiliencia al cambio climático y generar información verificable sobre sus avances. Durante la COP21 se anunció que Quito había conseguido la conformidad total de la iniciativa del Compact of Mayors, convirtiéndose así en una de las 45 ciudades del mundo que ha logrado este reconocimiento. Previa su aprobación, la coalición verificó las acciones de Quito frente al cambio climático y el cumplimiento de todos los requisitos: reporte de compromisos, inventario de huella de carbono, planes de mitigación y adaptación al cambio climático. Actualmente, el alcalde de Quito, Mauricio Rodas, es miembro del Comité Ejecutivo Regional del ICLEI.

A fin de poder observar las relaciones encontradas entre los eventos internacionales, nacionales y locales se presenta a continuación un diagrama que permite observar la secuencia histórica de los eventos y recapitular los hallazgos producto de la investigación realizada (figura 1).

FIGURA 1. SÍNTESIS HISTÓRICA DE LOS EVENTOS VINCULADOS CON EL IMPULSO DE UNA AGENDA AMBIENTAL QUE PROCURE EL DESARROLLO SOSTENIBLE EN QUITO

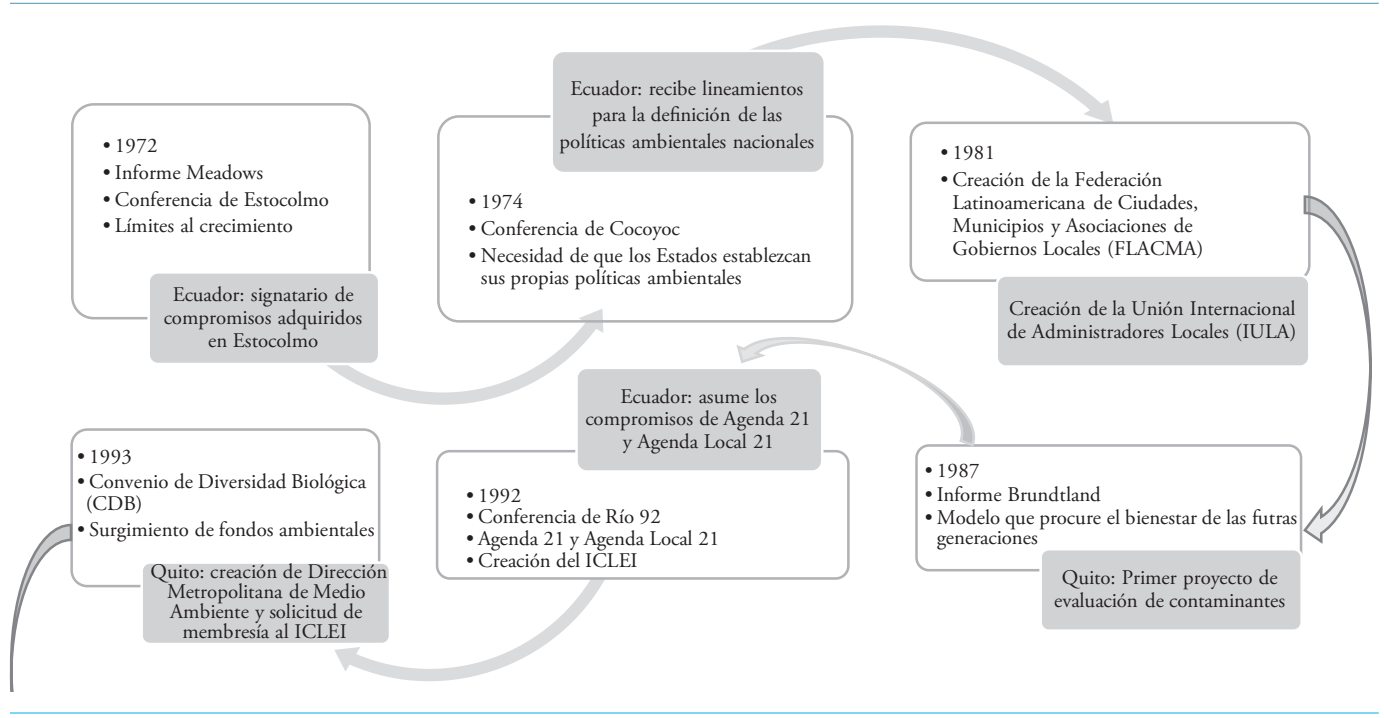




\section{CONCLUSIONES}

El reconocer que las relaciones sociedadambiente no son apolíticas lleva a cuestionarse cuáles son las motivaciones para que un tema vaya ganando espacio y se convierta en un pro- blema público susceptible de ser incluido en la agenda política. A partir de ello se intenta dilucidar cuáles son los factores que inciden para que un gobierno local asuma una agenda ambiental que procura la sostenibilidad. 
Un elemento que resalta es que entender la problemática de la política ambiental local obliga a contextualizar lo que sucede a nivel nacional e internacional y dejar de lado la concepción de que lo único que se debe analizar es la relación entre crecimiento poblacional y presión a los recursos naturales en un territorio.

Es innegable que el año 1972 constituye un punto crítico en la política internacional ambiental, pero es en la Conferencia de Río 92 cuando se definen acciones para aportar al modelo de sostenibilidad. Con base en los compromisos suscritos en la Agenda 21 y la Agenda 21 Local se reconoce la importancia de las ciudades en la procura del desarrollo sostenible y se impulsa una ola de acciones nacionales y locales a las cuales el Ecuador se sumó por ser signatario de estos compromisos.

Quito ha demostrado liderazgo no solo a nivel del país, sino también a nivel internacional para asumir una agenda que tome en cuenta los temas ambientales. La ciudad ha priorizado un modelo de sostenibilidad en el que resalta entre su prioridades la conservación de la biodiversidad y el manejo integrado de ecosistemas, también propende por convertirse en una ciudad verde y procurar la mejora de la calidad del aire, agua, suelo, protección de los recursos naturales y de la biodiversidad. Adicionalmente, se ha propuesto convertirse en una ciudad pionera en respuestas al cambio climático.

La investigación ha demostrado que Quito, capital del país, ha tenido un gran dinamismo en temas ambientales y se ha posicionado entre los primeros municipios que a nivel mundial han logrado acreditarse por cumplir los compromisos en temas de cambio climático. Se evidencia que los avances municipales son independientes de las acciones de otras instancias y otros niveles de gobierno. La fortaleza que ha ido asumiendo su aparataje administrativo por los años de especialización le ha dado experiencia para el abordaje de la temática.

La consolidación de la institucionalidad ambiental en el municipio de Quito data de 1994, cuando se crea la Dirección Metropolitana de Medio Ambiente y es casi media década después que se establece el primer Plan Maestro de Gestión Ambiental. Quien impulsó este documento fue un alcalde, con una amplia trayectoria y reconocimiento a nivel nacional e internacional por su experiencia como empresario, político pero también como ambientalista. Esto demuestra la relevancia que un líder tiene en estos procesos.

Aunque la normatividad nacional ha evolucionado desde la Constitución de 1998 favoreciendo el modelo de desarrollo sostenible, es en el año 2008 cuando se genera un hito al lograr el reconocimiento de los derechos de la naturaleza y de la ciudad a nivel constitucional, así como un nuevo modelo de planificación que procura el Buen Vivir. La puesta en marcha de estos temas motivó a la sociedad ecuatoriana a repensar el modelo de desarrollo. No obstante, analizar el liderazgo que Quito ha alcanzado en la temática permite evidenciar que los temas ambientales no fueron asumidos como respuesta a una nueva propuesta de desarrollo como la planteada en el marco del Buen Vivir, ni a cambios en la normatividad nacional o cambios constitucionales. Los avances que la ciudad ha tenido 
con respecto al posicionamiento del tema fueron previos a que el país lo priorizara en su Constitución.

Adicionalmente, se debe considerar que la Constitución del 2008 estableció un marco normativo para la descentralización de las funciones del Estado. En un modelo de descentralización, la definición de las competencias de gestión ambiental deben estar claramente establecidas para evitar conflictos por los roles y las funciones que deben desempeñar los diferentes niveles de Gobierno. Sin embargo, Quito no ha dependido de la normatividad para asumir sus funciones ya que incluso ha sobrepasado las competencias que le han sido asignadas. El asumir competencias ambientales requiere preparación y aprendizaje y, evidentemente, si un municipio ha venido trabajando en los temas podrá dinamizar la velocidad del cambio en su territorio.

La influencia del entorno internacional es incuestionable ya que cuando Quito comienza a formar parte de los espacios de debate internacional asume compromisos de liderazgo en los temas de sostenibilidad. Gracias al apoyo de redes globales especializadas en el tratamiento de la temática a las que el gobierno municipal se fue aliando empezaron a generase nuevos espacios de diálogo y discusión. A partir de esto se establece que para que un tema sea incorporado en la agenda pública debe ser posicionado como un problema público tanto a nivel internacional como nacional y local.

Marginalmente hablando, un Gobierno preparado potencializa sus ganancias cuando firma un nuevo convenio o se asocia a un nuevo proceso pues ha pasado la fase de aprendizaje y se encuentra en una etapa en la cual se involucra con temas que son nuevos a nivel global y en los que el conocimiento se va construyendo. Los gobiernos que son parte de esta dinámica se ubican en la cúspide del debate y es muy difícil que se vuelvan a rezagar pues los otros gobiernos tienen un largo camino que recorrer solo para ubicarse en su situación de partida.

La conformación de redes, coaliciones y grupos podría considerarse un punto crucial en la incorporación de los temas ambientales en la agenda de política pública. No obstante, la suscripción de convenios y la firma de acuerdos son insuficientes para generar acciones concretas y resultados verificables, la incidencia de estos procesos solo puede ser confirmada en la ejecución de las políticas.

Los eventos internacionales así como las redes y alianzas conformadas han dado la oportunidad a los gobiernos para que se vayan involucrando en la temática y generen capacidades para enfrentar los retos ambientales pero, como se dijo, no inciden en su puesta en marcha. En el marco de las redes globales es necesario mencionar también la capacidad de influencia de los delegados gubernamentales que participan en las reuniones organizadas. Una de las mayores ventajas que tiene Quito es que quienes asisten a estos eventos tienen poder de decisión y pueden trasladar los compromisos a acciones concretas.

Adicionalmente, se debe establecer el hecho de que si la máxima autoridad del gobierno local participa como representante de su institución en las redes globales y es reconocido como autoridad en dichos espacios, la posibilidad de asumir una agenda que promueve el desarrollo sostenible se potencializa. 
Lo antes expuesto permite confirmar que los temas ambientales no son incorporados de forma natural en la agenda de política pública, sino que son los factores endógenos y exógenos los que confluyen para permitir que ciertas condiciones ambientales sean asumidas y posicionadas como un problema de política público que debe ser atendido.

\section{REFERENCIAS}

Agenda 21 Local (1992). Recuperado de http://www. famp.es/recsa/Documentos/2_Agenda_21/A_ Agenda21_Local.pdf

Albán, M. A., Barragán, D., Bedón, R. y Muñoz, G. (2011). Ecuador ambiental 1996-2011: un recorrido propositivo. Quito: CEDA.

Alcaldía del Distrito Metropolitano de Quito (2011). Agenda Ambiental del Distrito Metropolitano de Quito 2011-2016. Quito: Secretaría de Ambiente.

Altmann, P. (2013). El Sumak Kawsay en el discurso del movimiento indígena ecuatoriano. Indiana 30, 283-299. Recuperado de http://www.iai. spk-berlin.de/fileadmin/dokumentenbibliothek/Indiana/Indiana_30/IND_30_2013_283299_Altmann

Cepal (2016). Declaración de Coyococ 1974. Recuperado de http://repositorio.cepal.org/bitstream/handle/11362/34958/S7800686_es.pdf?sequence=1

Cockburn, A. y Ridgeway, J. (1979). Political Ecology. New York: New York Times Books.

Constitución 1998 (2016). Constitución Política de la República de Ecuador de 1998. Recuperado de http://pdba.georgetown.edu/Constitutions/ Ecuador/ecuador98.html
Constitución 2008 (2016). Constitución Politica de la República de Ecuador de 2008. Recuperado de http://www.asambleanacional.gov.ec/documentos/constitucion_de_bolsillo.pdf

cootad (2016). Código Orgánico de Ordenamiento Territorial Autonomía y Descentralización 2010. Recuperado de http://virgiliohernandez.ec/ asamblea-wp/?p=2596.

Cop (2007). Conferencia de las Partes 13. Recuperado de http://unfccc.int/meetings/bali_dec_2007/ session/6265.php

De Castro, F., Hogenboom, B. y Baud, M. (2015). Gobernanza ambiental en América Latina. Buenos Aires: Consejo Latinoamericano de Ciencias Sociales.

De Munck, J. (2014). ¿Qué es una capacidad? Revista Latinoamericana de Politicas y Acción Pública, 1(1).

Duhau, E. (2001). Las metrópolis latinoamericanas en el siglo XXI: de la modernidad inconclusa a la crisis del espacio público. Cadernos Ippur, Rio de Janeiro, Xv (1).

Empresa Pública Metropolitana de Aseo (emaseo) (2010). Plan de servicios de aseo. Administración Zonal La Delicia. Quito: eMASeo.

Fischer, F.y Gottweis, H. (2012). The argumentative turn revisited: public policy as communicative practice. Durham: Duke University Press.

Fischer, F. y Miller, G. (2007). Handbook of Public Policy Analysis. Boca Raton: CRC.

FLACMA (2015). Federación Latinoamericana de Ciudades, Municipios y Asociaciones de Gobiernos Locales. Recuperado de http://www.flacma.com/index. php?option=com_content $\&$ view $=$ article $\&$ id $=$ 54\&Itemid $=59$.

FLACSO, MAE, PNUMA (2008). Geo Ecuador 2008. Informe sobre el estado del medio ambiente. Quito: FLACSO. 
FONAG (2016). Fondo para la protección del agua. Recuperado de http://www.fonag.org.ec/inicio/ index.php

Fondo de Población de las Naciones Unidas (UNFPA) (2007). Estado de la Población Mundial 2007. Liberar el potencial del crecimiento urbano. División de Información y Relaciones Externas del UNFPA. Recuperado de https://www.unfpa.org/ sites/default/files/pub-pdf/s

Fontaine, G., Narváez, I. y Cisneros, P. (2008). Informe sobre el estado del medio ambiente. Flacso. Recuperado de http://www.pnuma.org/deat1/ pdf/Ecuador\%20pdf/09.\%20Capitulo\%20 7.\%20Politicas\%20ambientales.pdf el 3 de enero del 2016.

Green-Pedersen, C. y Mortensen, P. (2013). Attention, politics and the public. En Araral, E., Fritzen, S., Howlett, M. R. y Wu, X. Routledge Handbook of Public Policy (167-174). Estados Unidos y Canadá: Routledge.

Harvey, D. (1996). Justice, nature and the geography of difference. Blackwell: Oxford.

Heynen, N., Kaika, M. y Swyngedouw, E. (2006). In the Nature of Cities. Urban political ecology and the politics of urban metabolism. London and New York: Routledge Taylor and Francis Group.

Hidalgo-Capitán, A. y Cubillo-Guevara, A. (2014). Seis debates abiertos sobre el Sumak Kawsay en Íconos. Revista de Ciencias Sociales. En busca del sumak kawsay (48). Recuperado de http:// www.google.com.ec/url?sa $=t \& r c t=j \& q=\& e s r c$ $=s \&$ source $=$ web $\& c d=1 \&$ ved $=0$ CBwQFjAA $\&$ url=http $\% 3 \mathrm{~A} \% 2 \mathrm{~F} \% 2 \mathrm{Fdialnet}$.unirioja.es $\% 2 \mathrm{~F}$ descarga\%2Farticulo\%2F4722037.pdf\&ei=c 0ViVearKYvRggSz9oHwBQ\&usg=AFQjCNF cB3_I7sU4i0m47xL3DMnucmeyVA\&sig2=sUwG5WD-9sJW-eOlgYddw\&bvm=bv.93
ICLEI (2015). Local Governments for Sustainability. Recuperado de http://www.iclei.org/es/iclei-global/ who-is-iclei/faq.html

Kingdon, J. (2003). Agendas, alternatives, and public policies. Reading: Addison-Wesley Educational Publishers Inc.

Krainer, A. y Mora, M. F. (2013). Actores, procesos y retos de la descentralización en Ecuador. Una mirada retrospectiva a la década 1998-2008. Quito: FLACSO.

Larrea, A. (2012). Modo de desarrollo, organización territorial y cambio constituyente en el Ecuador. Quito: SENPLADES.

Lezama, J. (2008). La construcción social y politica del medio ambiente. México: El Colegio de México, Centro de Estudios Demográficos y de Desarrollo Urbano.

PNUD-CISMIL-MDMQ (2008). Distrito Metropolitano de Quito: Objetivos de Desarrollo del Milenio. Estado de situación 2007. Quito: SENPLADEs/ PNUD/FLACSO.

PNuma, FamdmQ, flacso Ecuador (2011). Perspectivas del ambiente y cambio climático en el medio urbano: ECCO Distrito Metropolitano de Quito. Recuperado de http://www.flacsoandes.edu.ec/ libros/digital/54250.pdf

REDLAC (2016). Red de Fondos Ambientales de Latinoamérica y el Caribe . Recuperado de http://redlac.org/

Robbins, P. (2012). Political Ecology: a critical introduction (2 ed.). United Kingdom: Blackwell Publishing.

Secretaría Nacional de Planificación y Desarrollo (sENplades) (2007). Plan Nacional de Desarrollo 2007-2010. Quito: senplades. Recuperado de http://plan2007.senplades.gob.ec/

Secretaría Nacional de Planificación y Desarrollo (sENPLADES) (2015). Recuperado de http://plan2007. senplades.gob.ec/

OPERA, $\mathrm{N}^{\circ} 19 \cdot \mathrm{Julio-Diciembre} 2016 \cdot p \mathrm{p}$. 35-53 
Sistema de Naciones Unidas (1992). Agenda 21 Local. Recuperado de http://www.famp.es/recsa/Documentos/2_Agenda_21/A_Agenda21_Local.pdf UCLG (2016). Red Mundial de Ciudades y Gobiernos Localesy Regionales. Recuperado de http://www. worldmayorscouncil.org/members.html UNFPA (2007). Estado de la población mundial 2007. Liberar el potencial del crecimiento urbano. UNFPA.
Vallejo, R. (2009). Quito, de municipio a Gobierno local: innovación institucional en la conformación y gobierno del Distrito Metropolitano de Quito. Quito: flacso Ecuador.

World Mayors Council on Climate Change (s. f.). Recuperado de http://www.worldmayorscouncil. org/about/history.html 